\title{
Un estudio sobre la comprensión de términos espacio-temporales en niños de 4 a 6 años
}

\section{José Antonio Carranza* Alfredo G. Brito Gloria Torrecilla}

\author{
Universidad de Murcia
}

$\mathrm{L}$

A hipótesis de los rasgos semánticos ha sido una de las opciones para explicar la adquisición del significado de las palabras que ha originado un buen número de investigaciones y una enorme controversia en el campo de la psicolingüística.

Uno de los aspectos estudiados por este modelo es la adquisición de los términos espacio-temporales. En este sentido H. Clark (1973, pág. 57) sugería que «porque el tiempo es una metáfora espacial, el uso de un término que denote tiempo debe haber estado precedido por el uso del término comparable que denote espacio". Así pues, los pares de palabras «antes/después», «delante/detrás» y "primero/último» se adquiririan más pronto en su sentido espacial.

Algunas investigaciones apoyan esta hipótesis, mientras que otras ofrecen explicaciones opcionales incluyendo otros factores que no sean los estrictamente semánticos, tales como la complejidad cognitiva de la tarea (Goodz, 1982), la interpretación deíctica (Harris y Strommen, 1979) y el uso que la comunidad lingüistica hace de dichos términos ( $R$ ichards y Hawpe, 1981).
Goodz (1982) diseñó un experimento para separar los dos factores, complejidad semántica y complejidad cognitiva, que podrían incidir en la adquisición del par «antes/después». Los resultados de esta investigación apoyan que la estructura de la frase en las consignas es un determinante en la ejecución y que el orden gramatical inverso es más complejo, tal como sugieren otros autores Uohnson, 1975; Brown, 1977; Coker, 1978).

La deixis en la interpretación espacial ha sido objeto de muchos trabajos (Leehey, 1973; Kuczaj y Maratsos, 1975; Leehey y Carey, 1978; Harris y Strommen, 1979). En todos ellos se ha demostrado que los ragos de "frente» y «espalda" de los objetos facilitan la comprensión espacial. La ausencia de estos rasgos requiere que el sujeto utilice su propio cuerpo para establecer la relación con los objetos.

Diversos estudios Johnson y Slobin, 1979; Richards, 1979; Richards y Hawpe, 1981) han demostrado que el significado no deíctico se adquiere más pronto que el significado deíctico.

\footnotetext{
* Dirección de los autores: Facultad de Filosofía y Ciencias de la Educación. Sección de Psicología.
} Universidad de Murcia. 
Un modelo alternativo a la propuesto de H. Clark (1973) de que la adquisición evoluciona desde la comprensión espacial a la temporal, pero nunca en sentido contrario, es el presentado por Richards y Hawpe (1981) según el cual la evolución se configura a partir del uso que la comunidad lingüística hace de los términos.

Otra hipótesis derivada de los rasgos semánticos se basa en la adquisición más temprana de los términos positivos en los pares de antónimos con respecto a los negativos. El argumento que justifica esta predicción tiene su raíz en la aditividad de rasgos semánticos: el término negativo posee más rasgos que el término positivo $y$, por consiguiente, es más complejo.

En este sentido los datos son contradictorios: en algunas investigaciones los resultados apoyan la hipótesis (Ben, 1970; Donaldson y Wales, 1970; E. Clark, 1971, 1972). En otros estudios, por el contrario, no ha sido confirmada (Amidon y Carey, 1972; Eilers y cols., 1974; Coots, 1975; Kuczaj y Maratsos, 1975).

El propósito de este trabajo consistía en estudiar la comprensión de tres pares de antónimos espacio-temporales, «antes/después», "delante/detrás»y «primero/último», en niños de 4,5 y 6 años. La prueba consistía en la ejecución de una consigna en tres tipos de tareas:

- Espacial: alineamiento de objetos en el espacio.

- Espacio-temporal: alineamiento de objetos en el espacio determinando un orden en el tiempo.

- Temporal: sucesión de acontecimientos.

La hipótesis que presentamos se basa, en primer lugar, en que el significado adquirido más pronto será el significado dominante en la comunidad lingüística adulta. En segundo lugar, que las asimetrías en la comprensión de los binomios no son sistemáticas $\mathrm{y}$, por tanto, no se ajustan al modelo expliciativo de los rasgos semánticos. En tercer lugar, la ejecución será mayor en las pruebas que requieren una interpretación no deíctica, es decir, en las que se utilicen objetos con rasgos, así como en las consignas temporales cuyo orden de mención corresponde al orden de ejecución. Finalmente, consideramos que la ejecución aumenta con la edad.

\section{METODO}

\section{Estudio con adultos}

De acuerdo con nuestra primera hipótesis realizamos un estudio con adultos para comprobar cuál es el significado dominante en la comunidad lingüística.

La muestra estaba formada por 90 sujetos cada uno de los cuales definió dos términos que no eran antónimos. Se obtuvo un total de 180 definiciones, 30 para cada palabra.

Los resultados indicaban que el significado dominante del par «antes/después» es temporal $(73,3$ por 100$)$; para el par «delante/detrás», espacial (73,3 por 100), y. en «primero/último» hubo un porcentaje elevado de definiciones ambiguas (76,6 por 100$)$. Hemos considerado definiciones ambiguas las que hacen una referencia explícita a ambas dimensiones, espacio y tiempo, y aquellas cuyo contenido no es espacial ni-temporal.

\section{Estudio con niños}

Se utilizó una muestra de 180 niños de 4,5 y 6 años de edad elegidos al azar entre diversas escuelas de Murcia. Se formaron nueve grupos de 20 sujetos cada uno, tres grupos por edad, cada uno de los cuales realizó la prueba con un par de palabras. El número de varones y mujeres era aproximadamente igual en cada uno de los grupos.

Los materiales empleados son los siguientes:

- Ocho muñecos de plástico representando personajes de cuentos infantiles.

- Cuatro figuras geométricas tridimensionales de madera: estrella, círculo, cuadrado y triángulo.

- Una plataforma rectangular de madera de color blanco de $45 \times 9 \times 5 \mathrm{~cm}$. en cuya superficie hay ocho pequeños rectángulos pintados de color rojo.

- Un autobús de madera de $36 \mathrm{~cm}$. de largo.

- Un cubo de plástico.

- Una casa de madera de $40 \mathrm{~cm}$. de altura de cuya base sobresale una plataforma de $25 \mathrm{~cm}$. de largo quedando, así en forma de L. La casa tiene cinco puertas, y encima de cada una de ellas, un piloto que se enciende accionando un botón situado en la plataforma horizontal frente a cada puerta. 


\section{Tarea espacial}

En la tarea espacial se emplean la plataforma rectangular, los muñecos y las figuras geométricas. Consta de varias subtareas en función del tipo de objetos (muñecos o figuras) y de la posición de la plataforma (posición lateral en eje izquierda-derecha o posición perpendicular en eje frente-atrás).

En el par «delante/detrás» se eliminan las subtareas laterales, ya que por su misma estructura semántica estos términos se adaptan mejor a las relaciones frente-atrás.

El procedimiento es el mismo en todas las subtareas: se presenta una configuración con dos objetos y se pide al sujeto que coloque otros dos según la consigna. La configuración se cambia en cada ensayo.

Cada subtarea consta de seis ensayos, tres para cada palabra del par. Las subtareas se presentan al azar. Las consignas son "Pon $\mathrm{X}$ (antes, después, delante, detrás) de $Y_{\text {». }}$

El procedimiento general tiene algunas variaciones para el par «primero/último» debido a que estas palabras suponen posiciones absolutas en el espacio.

Para este par la plataforma consta sólo de siete «ranuras». Se colocan tres objetos fijos quedando un solo espacio vacio entre uno y otro. Se pide al sujeto que coloque un cuarto objeto. Las consignas son las siguientes:

"Pon $\mathbf{X}$ de forma que sea el primero».

"Pon $\mathrm{X}$ de forma que sea el último».

\section{Tarea espacio-temporal}

El material empleado en esta tarea es el mismo que en la tarea espacial (plataforma, muñecos y figuras), añadiendo el cubo y el autobús que sirven de objetometa y marcan el carácter temporal de esta tarea mixta. El cubo se utiliza en las subtareas con figuras geométricas, y el autobús, en las subtareas con muñecos.

Consta de cuatro subtareas en función del tipo de objetos y de la situación del objeto-meta (derecha o izquierda del sujeto). En todas la plataforma se sitúa en posición lateral.

El procedimiento es igual que en la tarea espacial: para los pares «antes- después» y ¿«delante/detrás" se presenta una configuración con dos objetos fijos; para «primero/último» la plataforma con sólo siete ranuras presenta tres objetos fijos. Junto' a la plataforma se sitúa el objeto-meta.

Las subtareas se presentan al azar y constan de seis ensayos, tres para cada palabra del par. Las consignas son las siguientes:

«Pon $\mathbf{X}$ de forma que llegue al cubobus (antes/después, delante, detrás) de $Y »$.

"Pon $\mathrm{X}$ de forma que llegue al cubobus el primero/último».

\section{Tarea temporal}

Para la tarea temporal se utiliza la casa de madera y cinco muñecos, tos cuales se colocan delante de cada una de las puertas.

La tarea consiste en que el sujeto pulse dos botones según el orden especificado en la consigna. Hay cuatro tipos de consignas, dos para cada palabra del par.: De ellas, una es «congruente», es deciř, el orden expresado corresponde al orden de ejecución; la otra es «incongruente»: el orden expresado es el inverso al orden de ejecución.

Ejemplo de consigna «congruente»: «Pulsa X e Y; pulsa X delante de $\mathrm{Y}$ ».

Ejemplo de consigna «incongruente»: «Pulsa X e Y; pulsa Y delante de X».

\section{Diseño experimental}

La dificultad metodológica que plantea la comparación entre las tareas y entre los pares de palabras no nos ha permitido realizar un análisis global con todos los datos y en su lugar, de acuerdo con nuestras hipótesis, hemos realizado análisis de varianza.

- Para cada par de palabras y grupo de edad.

- Para cada par de palabras, grupo de edad y tarea.

- Para cada par de palabras y tarea.

Realizamos también una significación de diferencias entre medias para cada par de palabras, grupo de edad y tarea.

\section{RESULTADOS}

Los resultados del primer grupo de análisis señalan diferencias significatiłas 
en la ejecución de las distintas tareas con cada par de palabras, excepto en «antes/ después» a los 6 años $F(3,24)=1,2 \mathrm{p}<$ .05 y «primero/último» a los 5 años $F(3,24)=2,38 p<.05$.

En el par «antes/después» existen diferencias significativas a los 4 años $\mathrm{F}(5,22)=$ $=5,97 \mathrm{p}<.01$ y a los 5 años $F(8,35)=$ $=8,50 \mathrm{p}<.001$.

En el par «delante/detrás» hay diferencias en la ejecución en todos los grupos de edad: 4 años $\mathrm{F}(8,35)=9 \mathrm{p}<.001 ; 5$ años $F(8,35)=9,66 \mathrm{p}<.001 ; 6$ años $\mathrm{F}(5,22)=7,95 \mathrm{p}<.01$.

En el par «primero/último» hay diferencias significativas a los 4 años $F(5,22)=$ $=7,78 \mathrm{p}<.01$ y a los 6 años $F(3,24)=$ $=4,13 \mathrm{p}<.05$.

La significación de diferencias en ejecución entre los dos miembros del par sólo ha resultado significativa en los siguientes contextos y grupos de edad:



Con respecto a las diferencias entre subtareas, en el par «antes/después», tarea espacial, los resultados indican que a los 4 y 5 . años las subtareas con muñecos han obtenido mejor ejecución que las subtareas con figuras geométricas, tales diferencias disminuyen a los 6 años. En la tarea espacio-temporal no existen diferencias en ningún grupo de edad. En la tarea temporal las mayores diferencias se registran entre «antes»-congruente $y$ «después»-incongruente, siendo el primer término el que ha obtenido las puntuaciones más altas. El segundo, por el contrario, tiene puntuaciones significativamente inferiores al resto incluso a los 6 años.

En el par «delante/detrás», tarea espacial, las diferencias más notables aparecen a los 4 años entre figuras geométricas y muñecos. A los 6 años desaparecen las diferencias significativas. En la tarea espacio-temporal no existen diferencias en ningún grupo de edad y en la tarea temporal sólo a los 6 años entre «delante»-congruente y "detrás»-incongruente, por un lado, $y$ «delante»-incongruente $y$ «detrás»-incongruente, por otro.

En el par "primero/último» las diferencias en la tarea espacial están en el grupo de 4 años entre subtareas con figuras geométricas y con muñecos. A los 5 y 6 años la ejecución se iguala. En la tarea espacio-temporal sólo hay diferencias en el grupo de 5 años. En la tarea temporal las mayores diferencias se centran entre "primero» y «último» con independencia $I I 4$ de la estructura congruente-incongruente.
Las elevadas puntuaciones en "primero» se observan a los 4 y 5 años; a los 6 años, sin embargo, la ejecución alcanza el nivel máximo en las cuatro consignas.

Las diferencias en ejecución entre los tres grupos de edad han resultado significativas en los tres pares de palabras. En el par «antes/después» existen diferencias entre los grupos de edad en los tres contextos: Espacial $F(7,83)=17,66$ $\mathrm{p}<.001$; Espacio-temporal $\mathrm{F}(7,83)=$ $=11,92 \mathrm{p}<.001$, y temporal $F(5,01)=$ $=6,7 \mathrm{p}<.01$.

En el par «delante/detrás» existen diferencias sólo en las tareas espacio-temporal $F(5,01)=7 \mathrm{p}<.01$ y Temporal $F(3,16)=3,36 p<.05$.

En «primero/último» la ejecución presenta diferencias notablemente significativas en las tres tareas: Espacial $F(7,83)=15,21 \mathrm{p}<.001 ;$ Espaciotemporal $F(7,83)=16,08 p<.001$; Temporal $\mathrm{F}(7,83)=15,6 \mathrm{p}<.001$.

\section{DISCUSION Y CONCLUSIONES}

Los términos que han sido objeto de nuestro estudio poseen en castellano la doble acepción espacial y temporal. La Real Academia Española define los términos "antes" y "después» como adverbios de tiempo y lugar que denotan prioridad y posterioridad, respectivamente, en el tiempo y en el espacio.

Los términos «delante» y «detrás» son adverbios de lugar cuya función es situar 
en el espacio. Se pueden emplear como adverbios de modo.

Los adjetivos "primero» y «último» están definidos en relación al tiempo, espacio, orden y jerarquía. "Primero» pertenece a la categoría de los numerales ordinales y puede actuar también como sustantivo o adverbio. "Ultimo», sin embargo, no tiene relación etimológica con los numerales, pero funciona semánticamente como los ordinales (Real Academia Española, 1970).

En este sentido, el estudio realizado con adultos ha demostrado que una de las acepciones es más utilizada en el lenguaje común. El uso dominante en cada uno de los términos coincide con los resultados obtenidos en lengua inglesa (Hodun, 1975; Richards y Hawpe, 1981): para el par «antes/después» la dimensión temporal es la dominante, en «delante/detrás» prevalece el sentido espacial y en el binomio "primero/último» no queda claramente definido.

Los resultados de las pruebas realizadas con los niños permiten establecer una jerarquía en la comprensión de los binomios en función del contexto:

\begin{tabular}{|c|c|c|}
\hline «delante/detrás» & $\begin{array}{l}4 \text { años } \\
5 \text { años } \\
6 \text { años } \\
4 \text { años } \\
5 \text { años } \\
6 \text { años } \\
4 \text { años } \\
5 \text { años } \\
6 \text { años }\end{array}$ & $\begin{array}{l}\text { - Temporal } \\
\text { - Espacio-temporal y espacial } \\
\text { - Temporal y espacio-temporal } \\
\text { - Espacial } \\
\text { (No existen diferencias en la ejecución entre tareas) } \\
\text { - Espacial } \\
\text { - Espacio-temporal y temporal } \\
\text { - Espacial y espacio-temporal } \\
\text { - Temporal } \\
\text { - Espacial y espacio-temporal } \\
\text { - Temporal } \\
\text { - Temporal } \\
\text { - Espacio-temporal y espacial } \\
\text { (No existen diferencias en la ejecución entre tareas) } \\
\text { - Temporal y espacio-temporal } \\
\text { - Espacial }\end{array}$ \\
\hline
\end{tabular}

En el par «antes/después» la comprensión temporal es previa a la espacial sirviendo de intermediario entre ambas dimensiones el contexto espacio-temporal. A los 6 años el par es comprendido con independencia del contexto en que se utilice.

En el par «delante/detrás» se comienza por la comprensión espacial incorporando más tarde el contexto espaciotemporal. Parece que a los 6 años existen todavía dificultades para determinar el significado en tareas temporales.

El par "primero/último» ha resultado mejor comprendido en tareas temporales, aunque a los 5 años no existen diferencias significativas entre los contextos y aparecen de nuevo a los 6 años. Estos resultados se deben a una progresiva evolución de los 4 a los 5 años, evolución que con- tinúa a los 6 años alcanzando el 100 por 100 de aciertos en. la tarea temporal, lo cual produce diferencias significativas con respecto a la tarea espacial (85 por 100).

Richards y Hawpe (1981) obtuvieron resultados similares en los tres pares de palabras. Del mismo modo, Friedman y Seely (1976) constataron que a los 3 años las puntuaciones más altas en las tareas temporales correspondian a los términos «antes/después» y «primero/último».

En cuanto a la asimetría entre los dos miembros del par, tal como planteamos en nuestra segunda hipótesis, se han constatado diferencias según el contexto, grupo de edad y el binomio, en favor del término positivo en las situaciones siguientes:

\begin{tabular}{lll} 
Binomio & Edad & Tarea \\
\hline «antes/después» & 4 años & Espacio-temporal y temporal \\
& 5 años & Temporal \\
«delante/detrás» & 6 años & Temporal \\
"primero/último" & 4 años & Espacial, espacio-temporal y temporal
\end{tabular}


En el resto de las tareas, en los diferentes grupos de edad, no se han encontrado diferencias significativas en cuanto a la prevalencia de la comprensión del término positivo, aunque en casi todos los casos la mejor ejecución corresponde a este término; por consiguiente, los datos sugieren que el modelo de Clark resulta inadecuado para explicarla.

La tercera hipótesis que planteamos se basaba en la influencia que pueden tener en -la ejecución las características de la tarea. Los datos obtenidos confirman nuestra predicción. Así, è cuanto a la tarea espacial, existen difèrencias significativas entre las subtaréas, diferencias que se centran, fundamentalmente, entre las que utilizan figuras coñ rasgos (muñecos) con respecto a las que utulizan figuras geométricas. Esto parèce indicar que los rasgos de "frente»'y "üespalda" de los objetos facilitan la comṕrensión de los términos espaciales.

En la tarea espacio-temporal parece no existir una jerarquía en complejidad entre las subtareas. El objèto-meta posibilita una interpretación nọ̀ deíctica en las pruebas con figuras geométricas lo cual hace que se asemejen en dificultad a las pruebas con muñecos.

En líneas generales nuestros resultados confirman la hipótesis de que la interpretación deíctica de los términos espaciotemporales es más compleja que la interpretación no deíctica, es decir, el niño ejecuta mejor las tareas que requieren situar un objeto en el espacio, de acuerdo con sus propios rasgos, que aquellas que implican tomar el cuerpo del sujeto como referencia en la interpretación espacial de los términos (Harris y Strommen, 1979; Kuczaj y Maratsos, 1975; Tanz, 1980).

En la tarea temporal, las estructuras incongruentes han resultado más difíciles que las congruentes. Algunos estudios han constatado que el orden gramatical inverso es notablemente más complejo (Smith y McMahon, 1970; H. Clark, 1976).

Finalmente hay que destacar la evolución en la comprensión de los tres binomios en todos los contextos a lo largo de los tres grupos de edad:

En resumen, los resultados de nuestro trabajo sugieren que, en adquisición de los términos espacio-temporales, los niños empiezan por comprender mejor el significado que la comunidad lingüística les atribuye, de tal manera que en aquellos pares de palabras en los que se observa un significado prevaleciente en las definiciones adultas, la jerarquía en la ejecución hasta su dominio comienza por aquella tarea que hace referencia al uso que mayoritariamente dicha comunidad les da. Asimismo, las tareas espaciotemporales parecen mostrarse como paso intermedio para abarcar las posibles dimensiones que estos términos tienen; en otras palabras, las situaciones en las que la posición espacial determina un orden temporal, facilitan la adquisición de la dimensión no dominante, sirviendo de puente conceptual entre los dos sentidos.

Pero, aunque esto pueda ser una particularidad relevante para su explicación, es importante que también tengamos en cuenta determinadas características inherentes a las situaciones en las que se utilizan dichos pares de palabras, de suerte tal que hemos podido observar cómo en unos casos la presencia de rasgos distintivos, en otros la estructuración congruente de la consigna, e incluso en otros la utilización de un referente externo provocan mejores comprensiones, $y$, por tanto, ejecuciones que cuando las mismas no están presentes.

Existe un caso en el que la muestra adulta define, en su mayoría, de forma ambigua un par de palabras, "primerol último", que resulta ser el binomio que mayor número de acepciones posee de los tres definidos, como vimos más atrás. Esta diversidad de sentidos parece estar en el centro de la irregularidad que hemos encontrado en la evolución entre los diversos grupos de edad.

En cuanto a la adquisición de los binomios, en «antes/después» el sentido temporal es el que parece adquirirse en primer lugar. Más tarde, el niño empieza a relacionar el «tiempo anterior» (antes) con una posición espacial más próxima al punto de llegada, y el «tiempo posterior» (después), con una posición más lejana al punto de llegada. Pero si bien en las diversas tareas los objetos con rasgos para Richards y Hawpe (1981) determinan posiciones espaciales de delante/detrás, que sirven de intermediario en la adquisición del par «antes/después» en su sentido 
espacial, en nuestro caso los datos sugieren que al no existir diferencias entre las subtareas (tarea espacio-temporal), no es la presencia de rasgos distintivos lo que determina el dominio del sentido espacial, sino que es el objeto-meta el que sirve tanto para establecer la sucesión temporal como la posición de delantedetrás de-todo tipo de objetos. Consecuentemente, el primer significado espacial de "antes/después» hace referencia, como también para estos autores, a una interpretación no deíctica, pero no en cuanto al uso de objetos con/sin rasgos, sino más bien basada en la presencia de un objeto meta como punto de referencia. A los 6 años se presentaron, en la tarea espacial, diferencias entre las subtareas que poseen objetos con/sin rasgos. Pero este resultado parece provenir de que el sujeto, al no disponer de un objeto-meta de referencia, que tenía en la situación espacio-temporal, y al no dominar la interpretación no-deíctica, tiene que recurrir a la búsqueda de un elemento referencial externo a él, que en este caso recae en la presencia/ausencia de rasgos. Así pues, el orden de adquisición del par sería: Temporal-Espacio temporal-Espacial (objetos con rasgos)Espacial (objetos sin rasgos).

En el par «delante/detrás», a la inicial comprensión espacial le sigue la espaciotemporal, pero como acabamos de explicar, su comprensión supone una transferencia de los rasgos frente/espalda, no de los objetos con rasgos, sino, por contra, del objeto-meta, de manera tal que se produce la siguiente analogía: «delante»= $=$ «al frente de», indicando una posición anterior en el tiempo hacia la meta; "detrás» = «a la espalda de», implicando una llegada posterior a la meta. Posteriormente se adquiere el significado temporal, quedando el orden de adquisición del modo siguiente: Espacial-Espacio temporal-Temporal.

Con respecto al par "primero/último», en principio se observa una mejor ejecución en la dimensión temporal. En este binomio, el contexto espacio-temporal no parece actuar de puente conceptual entre las dimensiones temporal y espacial. El aumento generalizado con la edad en la ejecución de las tareas, nos hace pensar que su dominio se adquiere de forma independiente más que por analogía, como sucedía en los pares anteriores.

En definitiva, los resultados de nuestro trabajo pueden incluirse en la línea-de los estudios sobre el lenguaje espaciotemporal, que han señalado que la hipótesis de Clark, según la cual la significación temporal está necesariamente precedida de la significación espacial, no es consistente. En este sentido, consideramos que es necesario tener en cuenta factores tales como el signifcado que la comunidad lingüística confiere a estos términos, así como variables situacionales, para alcanzar una mejor comprensión respecto a la adquisición y dominio de los términos espacio-temporales.

\section{Referencias}

AmIDON, A., y CAREY, P.: “Why five years olds cannot understand 'before' and 'after'". Journal of Verbal Learning and Verbal Behavior, 1972, 11, 417-23.

BEM; S. L.: "The role of comprehension in children's problem solving". Developmental Psychology, 1970, 2, 351-358.

Clark, E.: "On the acquisition of the meaning of 'before' and 'after'". Journal of Verbal Learning and Verbal Behavior, 1971, 10, 266-275.

CI.ARK, E.: "On the child's acquisition of antonyms in two/semantic fields". Journal of Verbal. Learning and Verbal Bebavior, 1972, 10, 266-275.

Clark, H.: "Space, time,'semantics, and the child». En T. E. Moore (ed.): Cognitive development and the acquisition of language. Nueva York: Academic Press, 1973.

COOTS. J. H.: "Polarity and complexity in spatial adjective acquisition". Paper presented at the meetin of the Society for Research in child development, Denver, abril, 1975.

COKER, P. L.: "Syntactic and semantic factors in the acquisition of 'before' and 'after'". Journal of child 3 Lanğuage, 1978, 5, 261-277.

Donaldson, M., y WALES, R. J.: "On the acquisition of some relational teras". En J. R. Hayes (ed.): Cognition and the development of language, Nueva York, 1970.

Eilers, R. E.; Kimbrough Oller, D., y Ellington, J.: "The acquisition of word meaning for dimensional adjetives: the long and the short of it". Journal Child Language, 1974, 1, 195-204. 
FrenCH, L. A., y Brown, A. L.: «Comprehension of 'before' and 'after' in logical and arbitrary sequencens». Journal of child Language, 1977, 4, 247-256.

Friedman, W. J., y Seely, P. B.: «The child's acquisition of spatial and temporal word meaningsn. Child Development, 1976, 47, 1103-1108.

GoOD, N. S.: «Is Before Really Easier to Understand than After?» Cbild Development, 1982, 53, 822-825.

HARRIS, L. J., y StrommEN, E. A.: «The role of front-back features in children's 'front', 'back', and 'beside' placements of objects". Merrill Palmer Quarterly, 18, 259-271, 1972.

HoDUN, A.: «Comprehension and the developmeat of spatial and temporal sequence terms*. Tesis doctoral no publicada. Universidad de Wisconsin, 1975.

JoHNSON, H. I.: *The meaning of 'before' and 'after' for preschool children*. Joumal of Experimental Child Psychology, 1975, 19, 88-89.

JohnSTON, J. R., y SLOBIN, D. J.: “The development of locative expresions in English, Italian, SerboCroatian and Turkish.. Joumal of Child Language, 1979, 6, 529-545.

Kuczjaj II, S. A., y Maratsos, M. P.: «On the acquisition of front, back and side». Child Development, 1975, 202-210.

LEEHEY, S. C.: «The child's lexical and conceptual knowledge of front and back». Investigación no publicada, 1973.

LeeHey, S. E., y Carey, S.: «Up front: The acquisition of a concept and a word». Presentado al 10th Child Language Research Forum, Standford, California. Abril, 1978.

Real Academia de la Lengua, 1970.

RICHARDS, M. M.: «Sorting ont what's in a word from what's not: Evaluating Clark's semantic features acquisition theory". Journal of Verbal Learning and Verbal Behaviorn, 1976, 15, 655.

RiCHARDS, M. M., y HAwZE, L. S.: \&Contrasting patterns in the acquisition of spatial/temporal terms». Joumal of Experimental Cbild Psychology, 1981, 32, 485-512.

SMITH, K. H., y MCMAHON, L. E.: «Understanding order information in sentences: some recent work at Bell Laboratories». En G. Flores diAreais y W. J. M. Levelt (eds.) Advances in psycbolinguistics. Amsterdam: North-Holland, 1970.

TANZ, C.: "Studies in the acquisition of deictic expressions». Cambridge Studios in Psycholinguistics, 26, Cambridge University Press, 1980.

\section{Resumen}

Este estudio trató de comprobar si la comprensión de los pares de palabras. *antes/después", «delanteldeträs* y aprimerolkéltimo* presenta relación con el significado dominante en la comunidad lingüistica, la complejidad de la carea, la asimetria de los términos y la edad. Se utilizaron 90 adulios y 180 niños entre 4 y 6 años. Los adultos definieron las palabras a analizar; los niños ejecutaron las consignas en tres contextos: espacial, espacio-temporal y semporal. Los resultados indican que el par a antes/después * es comprendido más pronto en el sentido temporal; el par - delante ldetrás: en sentido espacial. Estos datos coinciden con los obrenidos en las definiciones adultas. «Primera siltimo* es mejor comprendido en tareas temporales, aunque los adiltos dieron definiciones ambiguas. Por otra parte, la compejidad de la tarea parece influir en la comprensión: la ausencia de rasgos de los objetos y: el. orden gramatical inverso dificultan la tarea. Estos resultados sugieren que la bipótesis de los rasgos semánticos es insuficiente para explicar la adquisición del signifcado.

\section{Summary}

This study investigated whether the comprebension of the following pairs of words cbeforelafter, "abeadbehind" and afirstllast" could be related to their dominant usage in linguistic community, complexity of task, asymmetry between terms and age. 90 adults and 180 children (4-5 years old) were used. Adults defined words to discover the dominant sense. Children performed tasks in three contexts: spatial, spatial-temporal, temporal. Results show tbat abeforelafters is understood at earlier age in a temporal sense; abeadibehind" in a spatial sense; " firstllast. is better understood in a semporal context, altbough ibe adults definitions were ambiguous. Complexity of task seems influence in comprebension: absence of features in abjects and inversal gramatical construction make the task difficult. Results suggest that the Semantic Feature Hypothesis is invalid to account for the acquisition of meaning.

\section{Résumé}

Cet issude essaie de montrer si la comprébension des paires de mots *avantlapris». adevant/derrièren es "premier/ dernierw a des rapports avec la signification dominante dans la comsmunauté linguistique, la complexité de la táche, lasymetrie des temes et l'age. On a pris 90 adultes et 180 enfants de 4 à 6 ans. Les adultes ont défini les mots.à 
analyser; Les enfants ont executé les consignes en trois contextes: spatial, spatia-temporel et temporel. Les résultats montrent que la paire acuantlaprès* est comprise plus tôt en un sens temporel; la paire "devant/derrière" en un sens spatial. Ces données s'accordent avec ceux obtenus chez les adultes. «Premierldérnier est mieux compris en tâches temporelles si bien les adultes ont foumi des definitions ambigües. D'ailleurs, la complexité de la tâche semble influencer sur la comprebension: labsence de traits des objets et l'ordre grammatical inverse rendent la tâche difficile. Ces résultats suggerent que l'bypotbèse des traits semantiques est insuffisante pour expliquer l'acquisition de la signification. 